\title{
Structure determination, thermal stability and dissolution rate of $\delta$-indomethacin
}

Iryna Andrusenko ${ }^{\dagger}$, Victoria Hamilton ${ }^{\ddagger}$, Arianna E. Lanza ${ }^{\dagger}$, Charlie L. Hall ${ }^{\ddagger}$, Enrico Mugnaioli ${ }^{\dagger}$, Jason Potticary ${ }^{\ddagger}$, Asma Buanz ${ }^{\S}$, Simon Gaisford ${ }^{\S}$, Anna M. Piras ${ }^{\searrow}$, Ylenia Zambito ${ }^{*, \diamond}$, Simon R. Hall ${ }^{*, \neq}$, and Mauro Gemmi ${ }^{*, \dagger}$

†Center for Nanotechnology Innovation@NEST, Istituto Italiano di Tecnologia, Piazza San Silvestro 12, Pisa 56127, Italy

‡School of Chemistry, University of Bristol, Cantock's Close, Bristol BS8 ITS, UK

§UCL School of Pharmacy, University College London, 29-39 Brunswick Square, London WC1N 1AX, UK

${ }^{\bullet}$ Department of Pharmacy, University of Pisa, Via Bonanno 33, Pisa 56126, Italy

KEYWORDS: electron diffraction; structure determination; pharmaceutical compounds; polymorphism;

bioavailability; indomethacin; non-steroidal anti-inflammatory drugs

\begin{abstract}
The structure solution of the $\delta$-polymorph of indomethacin was obtained using threedimensional electron diffraction. This form shows a significantly enhanced dissolution rate compared with the more common and better studied $\alpha$ - and $\gamma$-polymorphs, indicating an increased bioavailability for medicinal applications. The structure was solved in non-centrosymmetric space group $P \mathbf{2}_{1}$ and comprises two molecules in the asymmetric unit. Packing and molecule conformation closely resemble indomethacin methyl ester and indomethacin methanol solvate. Knowledge of the structure allowed the rational interpretation of spectroscopic IR and Raman data for $\delta$-polymorph and a tentative interpretation for still unsolved indomethacin polymorphs. Finally, we observed a solid-solid transition from $\delta$-polymorph to $\alpha$ polymorph that can be driven by similarities in molecular conformation.
\end{abstract}

\section{INTRODUCTION}

Indomethacin (IMC) is a non-steroidal anti-inflammatory drug (NSAID) with high degree of antiinflammatory, analgesic and antipyretic activities. ${ }^{1-3}$ It was first discovered in $1963^{4}$ and since then has been extensively studied in clinical trials as one of the most effective NSAIDs commonly used in treatment of migraine, arthritis and acute pain. ${ }^{5}$ It was also shown that IMC potentially has a potent direct antiviral activity against coronaviruses. ${ }^{6,7}$ 


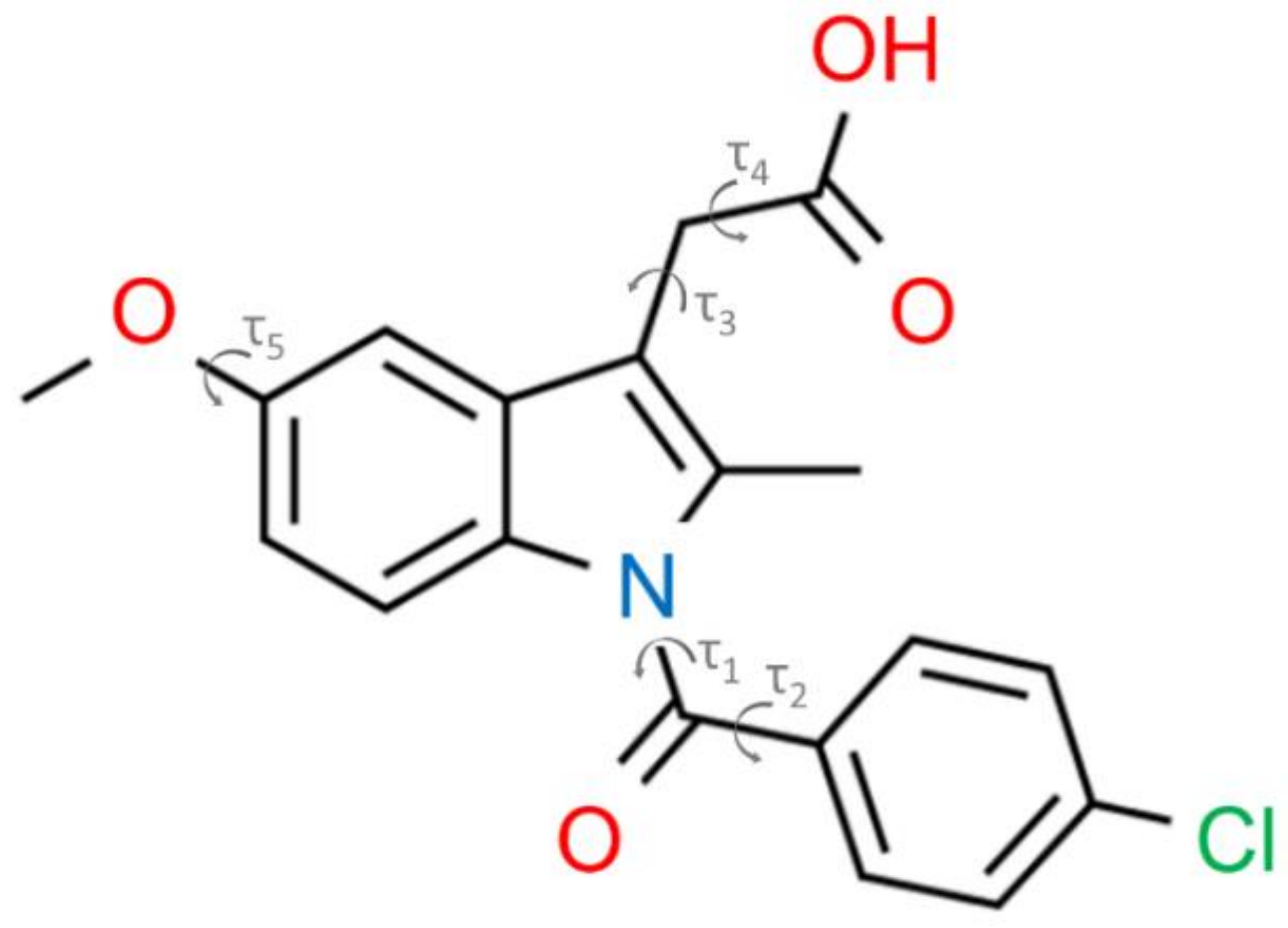

Figure 1. Molecular diagram of indomethacin, $\mathrm{C}_{19} \mathrm{H}_{16} \mathrm{ClNO}_{4}$. Free torsion angles are indicated.

IMC, namely 1-(4-chlorobenzoyl)-5-methoxy-2-methyl-1H-indole-3-acetic acid (Figure 1), is a weak acid classified by the Biopharmaceutical Classification System in class II (BCS class II). Drugs belonging to this class are easily permeable through mucous membranes, but are poorly soluble. Thus, IMC shows low bioavailability and, to be effective, requires special manufacturing and increased dosage, which in turn may worsen adverse effects such as gastric irritation or renal malfunctioning. ${ }^{3,8}$ Schemes for enhancing IMC dissolution rate and intestinal absorption include the crystallization of IMC as co-crystals, ${ }^{8-12}$ salts $^{13}$ and solvates, ${ }^{14,15}$ or its administration together with physiologic buffers or emulsions that modify the local $\mathrm{pH} .{ }^{16,17}$ The aqueous solubility of IMC is mainly driven by the ionization of its carboxylic group and hence it is freely soluble at $\mathrm{pH}>4 \cdot 5{ }^{18}$

IMC shows rich polymorphism. A comprehensive review about old and new pure-form polymorphs is reported in Surwase et al. ${ }^{19}$ Another form, $\tau$-indomethacin, was described by Van Duong et al. ${ }^{20}$ Despite the intensive investigation about IMC, only the crystal structures of the two most common forms are known: $\alpha$-IMC ${ }^{21}$ and $\gamma$-IMC. ${ }^{22}$ The $\gamma$-polymorph is commonly considered the thermodynamically stable form at ambient conditions, while the $\alpha$-polymorph is the most commonly observed among the metastable forms, often obtained as a first product after desolvation at high-temperature. ${ }^{14,23}$ However, a recent study, based on spectroscopic evidence and ab-initio molecular dynamics simulations, suggested instead that the $\alpha$-IMC is the thermodynamically stable form within the solid state, but its formation is hindered by a significant kinetic barrier. ${ }^{24}$ Almost all chemical, physical and theoretical studies about IMC have considered only these two forms, while very little is known about the others. This shortcoming poses critical limitations for the overall understanding of the IMC energy landscape and its medicinal applications.

Polymorphism is indeed a common aspect for most commercially relevant drugs. One-third of crystalline organic molecules and about half of marketed active pharmaceutical ingredients (APIs) are known to form polymorphs. ${ }^{25,26}$ The characterization of all polymorphic species and the understanding of the overall 
polymorphic energy landscape represents a prominent aspect of drug development and is crucial to establish efficacy, formulation and shelf-life. Moreover, the discovery of new polymorphs with different chemical and physical properties may result in treatments that are more effective and with reduced sideeffects. ${ }^{27}$

Here, we report the crystallization, structure determination and dissolution behavior of $\delta$-IMC, a poorly studied polymorph first mentioned almost 50 years ago $^{28}$ and whose structure has remained hitherto unknown. Pure $\delta$-IMC was obtained via desolvation of the methanol solvate form, and appears stable in the fridge for several months. Its crystallization results in fibrous crystals which are too small for conventional single-crystal X-ray diffraction (XRD). Structure determination of $\delta$-IMC was therefore obtained by three-dimensional electron diffraction (3D ED), ${ }^{29}$ performed in a transmission electron microscope (TEM) equipped with a single-electron detector..$^{3^{\circ}}$ In recent years, this protocol proved to be effective for structure determination of pharmaceutical compounds $\mathrm{s}^{31-34}$ and allowed the solution of a number of structures that could not be addressed by standard XRD. ${ }^{35-37}$ Determining the crystal structure of $\delta$-IMC delivered crucial information for understanding the remarkably high dissolution rate of this poorly-known polymorph, which appears now as a promising candidate for medicinal application.

\section{EXPERIMENTAL SECTION}

Crystallization. The $\delta$-IMC polymorph was obtained according to Crowley \& Zografi..$^{8}$ First, a saturated methanol solution was prepared at $60^{\circ} \mathrm{C}$, which was hot-filtered using a pre-heated $0.2 \mu \mathrm{m}$ PTFE filter attached to a $5 \mathrm{~mL}$ syringe. The methanol solution was then poured into a petri dish, covered with Parafilm and left to evaporate at room temperature (RT). After the IMC-methanol solvate crystallized, it was desolvated in a $\mathrm{SiO}_{2}$ desiccator under a rotary vane vacuum pump (Edwards $\mathrm{RV}_{3}, 10^{-3} \mathrm{mbar}$ ) at $\mathrm{RT}$ for 1014 days. The crystallinity of the sample was ascertained using an optical microscope with cross-polarized filters.

The commercially available $\gamma$-IMC polymorph (Sigma, $\geq 99 \%$ ) was used as received with no further purification. The metastable $\alpha$-IMC polymorph was crystallized according to Borka. ${ }^{28}$ This was achieved by forming a saturated solution of IMC in lab grade ethanol at $75{ }^{\circ} \mathrm{C}$. The solution was hot-filtered, as described for $\delta$-IMC. RT distilled water was subsequently added to the hot saturated solution which acted as an antisolvent and led to the precipitation of $\alpha$-IMC crystals; the resulting crystals were filtered and dried under ambient conditions.

Electron Crystallography. High-angle annular dark-field scanning transmission electron microscopy (HAADF-STEM) imaging and 3D ED data were recorded with a Zeiss Libra 120 TEM operating at $120 \mathrm{kV}$ and equipped with $\mathrm{LaB}_{6}$ source. $3 \mathrm{D}$ ED was performed in STEM mode after defocusing the beam in order to have a parallel illumination on the sample, as described by Lanza et al. ${ }^{39}$ ED patterns were collected in Köhler parallel illumination with a beam size of about $150 \mathrm{~nm}$ in diameter, obtained using a $5 \mu \mathrm{m} \mathrm{C}_{2}$ condenser aperture. Data were recorded by a single-electron ASI MEDIPIX detector..$^{30,31}$ The extremely lowdose illumination allowed data to be acquired at RT without evidence of sample amorphization under the electron beam.

Continuous data collections were performed while the TEM goniometer was rotating at a constant angular speed..$^{0,40,41}$ Due to mechanical drift, individual data sets covered a tilt range of only $10^{\circ}-40^{\circ}$. However, multiple data collections from the same crystal could be merged, reaching total angular ranges up to $120^{\circ}$.

$3 \mathrm{D}$ ED data were analyzed using the software PETS. ${ }^{42}$ Structure determination was achieved by simulated annealing (SA) ${ }^{32,37}$ as implemented in the software SIR2014. ${ }^{43}$ The resolution limit was set to 1.o $\AA$. The molecular model (Figure 1) was deduced from known polymorphic forms reported in the Cambridge 
Structural Database. ${ }^{44}$ Each of the two independent molecules of the structure has five free torsion angles and six translation/rotation parameters. Thus, the total number of parameters to be determined is twentytwo. No anti-bump restraint was used.

Data were treated with a fully kinematical approximation, assuming that $I_{\text {hkl }}$ was proportional to $\left|F_{\text {hkl }}\right|^{2}$. The model determined by SA was later refined with least-squares procedures embedded in the software SHELXL. ${ }^{45}$ Geometrical ties were added stepwise to check the consistency of the model. All hydrogen atoms were generated in geometrically idealized positions.

Powder X-ray Diffraction. Powder XRD data were acquired in Debye-Scherrer geometry using a STOE Stadi P diffractometer equipped with $\mathrm{Cu}-\mathrm{K}_{\alpha 1}$ radiation $(\lambda=1.5406 \AA)$, a Ge (111) Johansson monochromator from STOE \& Cie and a MYTHEN2 $1 \mathrm{~K}$ detector from Dectris. The sample was loaded in a borosilicate glass capillary ( $0.8 \mathrm{~mm}$ external diameter) and data were acquired in the range $2-62^{\circ} 2 \theta$ (maximum resolution ca. $1.5 \AA$ ) with an interval of $0.015^{\circ}$ between consecutive points. The unit cell and structural parameters were refined with Jana20o6..$^{4}$ The powder XRD pattern suffers from limited diffraction resolution and severe peak overlap due to the low symmetry and to the similarity between the lattice parameters $a$ and $c$. These limitations prevented a free Rietveld refinement, hence both molecules were modelled as semi-rigid bodies, restraining most interatomic distances and angles to geometrically idealized values, while enabling the free refinement of torsion angles.

Spectroscopy. Infrared (IR) spectroscopy data were acquired from $\alpha$-IMC, $\gamma$-IMC and $\delta$-IMC with a Perkin Elmer Spectrum Two FT-IR Spectrometer on solid powder. Raman spectroscopy was carried out using a Renishaw 2000 using a green $514 \mathrm{~nm}$ laser excitation with a spot size of $\sim 0.5 \mu \mathrm{m}$. Data were recorded at $2 \mathrm{~s}$ per acquisition with 10 acquisitions at $10 \%$ power of the system maximum $(\sim 8 \mathrm{~W})$, to achieve a resolvable signal-to-noise ratio and minimal damage to sensitive organic sample.

Experimental wavenumbers for $\mathrm{C}=\mathrm{O}$ stretching modes for $\varepsilon$-IMC, $\zeta$-IMC and $\eta$-IMC were taken from Surwase et al. ${ }^{19}$ and for $\tau$-IMC from Van Duong et al. ${ }^{20}$

Thermal Analysis. Simultaneous powder XRD and Differential Scanning Calorimetry (synchrotron powder XRD-DSC) experiments were performed at the Diamond Light Source using the Joint Engineering, Environment and Processing (JEEP) Beamline I12. A Q20 differential scanning calorimeter (TA Instruments) equipped with a refrigerated cooling system (RCS) was mounted onto the sample stage in the experimental area. Both apparatuses had been previously modified to allow the synchrotron beam to pass through. A $5 \mathrm{~mm}$ entry and $10 \mathrm{~mm}$ exit holes were drilled into the RCS, and a $3 \mathrm{~mm}$ entry and $5 \mathrm{~mm}$ exit holes into the DSC furnace. The X-ray beam had a monochromatic wavelength of $0.234 \AA$, and a diameter of $0.5 \mathrm{~mm}$. A Pilatus $2 \mathrm{M}$ CdTe detector was fitted $2 \mathrm{~m}$ behind the sample. Both the sampledetector distance and beam wavelength had been calibrated with cerium dioxide $\left(\mathrm{CeO}_{2}\right)$, using the calibration method stated in Hart et al. ${ }^{47}$ Patterns were recorded by collecting data for $4 \mathrm{~s}$, with a $2 \mathrm{~s}$ pause. The sample ( $9 \mathrm{mg}$ ) was hermetically sealed in a Tzero aluminum pan. The DSC was calibrated before for cell contact and enthalpy using indium standard (melting onset temperature $=156.6 \pm 0.5{ }^{\circ} \mathrm{C}$, enthalpy $=$ 28.72 $\mathrm{J} \mathrm{g}^{-1} \pm 3 \%$ ), according the manufacturer's instructions. DSC measurements were performed at a heating ramp rate of $10{ }^{\circ} \mathrm{C} / \mathrm{min}$ from $40{ }^{\circ} \mathrm{C}$ to $175{ }^{\circ} \mathrm{C}$ and one powder pattern was recorded per degree centigrade. Two aluminum hermetic lids were used to raise the pans inside the DSC furnace to center the sample in the beam direction; these pans were also included in the calibration. A nitrogen purge gas (50 $\mathrm{mL} / \mathrm{min}$ ) was used throughout. Data were collected with TA Instruments Advantage software and initially analyzed with TA Universal Analysis software. DAWN Science Workbench was first used to mask regions of unrepresentative spots of high intensity in the $2 \mathrm{D}$ Pilatus data caused by large grain/crystal size of the samples. The same software was used to convert the $2 \mathrm{D}$ data into $1 \mathrm{D}$ diffraction patterns. 
Dissolution. To determine the intrinsic solubility of the polymorphs $\alpha$-IMC, $\gamma$-IMC and $\delta$-IMC, an excess of each powdered polymorph was dispersed in simulated gastric fluid (SGF, $\mathrm{HCl}$ o.1 M) and the dispersion was stirred in an oven at $37^{\circ} \mathrm{C}$ for 96 hours, after which thermodynamic equilibrium was reached. The solution was then analyzed with a Perkin Elmer Lambda 25 UV spectrophotometer at a wavelength of 263 $\mathrm{nm}$ after filtration through a cellulose acetate filter $(0.45 \mu \mathrm{m})$.

To determine the dissolution rate of polymorphs $\alpha$-IMC, $\gamma$-IMC and $\delta$-IMC, the powders were compressed into flat-faced tablets of $1.2 \mathrm{~mm}$ diameter and $200 \mathrm{mg}$ weight, with a hydraulic press, applying a force of $4000 \mathrm{~kg}$ for 5 minutes. Tablets consisted of $180 \mathrm{mg}$ of lactose:starch 8:1 and $20 \mathrm{mg}$ of each polymorph previously ground in a mortar. ${ }^{13}$ Each tablet was subjected to the dissolution test as reported in the European Pharmacopoeia 1oth edition (Ph. Eur. 10.0), using an Erweka light paddle dissolution apparatus. A volume of $750 \mathrm{~mL}$ of SGF was placed in the dissolution vessel and, as the temperature of $37^{\circ} \mathrm{C}$ was reached, the tablet under test was introduced in the vessel and stirring at $150 \mathrm{rpm}$ was started. After stirring for two hours, $250 \mathrm{~mL}$ of $0.20 \mathrm{M}$ trisodium phosphate solution was added and the $\mathrm{pH}$ was adjusted to 6.8 with $\mathrm{NaOH} 2 \mathrm{M}$. The resulting dissolution medium was referred to as simulated jejunal fluid (SJF). At predetermined time intervals, $10 \mathrm{~mL}$ of dissolution medium was replaced with $10 \mathrm{~mL}$ of fresh medium, prethermostated at $37^{\circ} \mathrm{C}$, and analyzed for IMC after filtration, as described above. With each polymorph the assay was carried out in triplicate. The Kruskal-Wallis non-parametric statistical test was used to compare the dissolution profiles.

Lactose, starch, hydrochloric acid, trisodium phosphate dodecahydrate and sodium hydroxide were all purchased from Sigma-Aldrich.

\section{RESULTS AND DISCUSSION}

Structure solution and refinement. Growth of $\delta$-IMC via desolvation resulted in semi-transparent, long, flexible and intertwined wires (Figure 2A), which showed bright polychromatic scattering when illuminated under polarized light (Figure $2 \mathrm{~B}$ ). Some wires were isolated and gently crushed (Figure $2 \mathrm{C}$ ) before being dispersed on a carbon-coated Cu TEM grid, without the use of any solvent or sonication. 

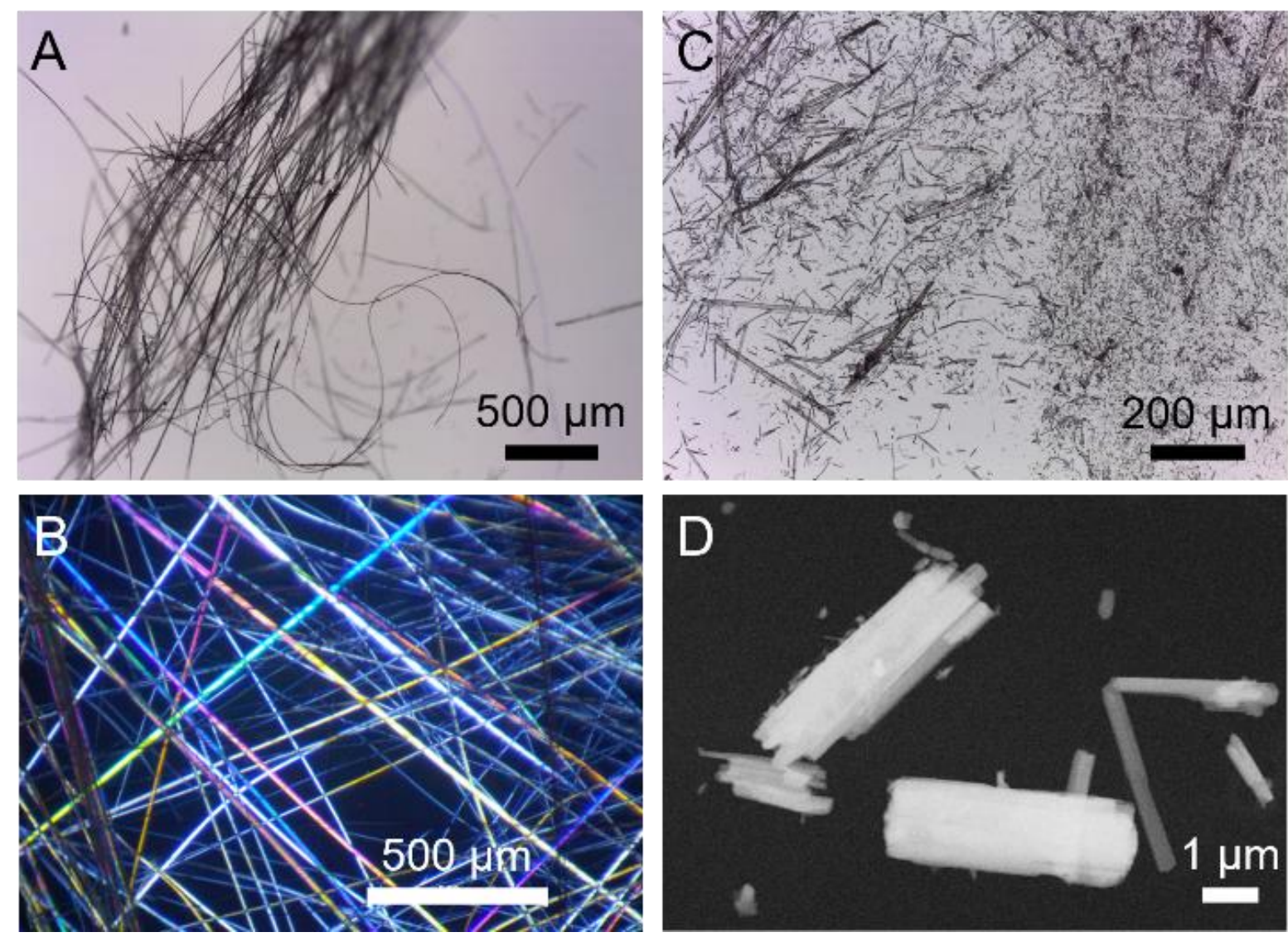

Figure 2. Optical images of $\delta$-IMC under white light before (A) and after (C) crushing. Optical image of $\delta$ IMC under polarized light, showing birefringent scattering (B). HAADF-STEM image of $\delta$-IMC after crushing and deposition on the TEM grid (D).

$3 \mathrm{D}$ ED data were recorded from twenty $\delta$-IMC crystal fragments of different sizes (Figure $2 \mathrm{D}$ ). All $3 \mathrm{D}$ ED data sets were consistent with a primitive monoclinic cell with approximate parameters $a=18.4 \AA$, $b=5.0$ $\AA, c=18.4 \AA, \beta=95.8^{\circ}$. Cell parameters were refined and validated with a LeBail fitting against powder XRD, from which the following refined lattice parameters were obtained: $a=18.3195(5) \AA, b=5.09993(9)$ $\AA$, $c=18.5386(5) \AA, \beta=95.771(2)^{\circ}$. Such a cell would conveniently host four IMC molecules. Upon inspection of the reciprocal space reconstruction, the reflection rule oko: $k=2 n$ was identified, hence suggesting a crystallization in the centrosymmetric space group $P \mathbf{2}_{1} / m$ (11), with one independent IMC molecule in the asymmetric unit, or in the non-centrosymmetric space group $P 2_{1}$ (4), with two independent IMC molecules in the asymmetric unit.

Structure solution was performed by SA using the most complete ${ }_{3} \mathrm{D}$ ED data set that showed no evidence of polycrystallinity or mosaicity. Structure solution attempts in space group $P 2_{1} / m$ proved unstable and could not be refined. Conversely, a sound structural model could be determined by reducing the symmetry to space group $P 2_{1}$.

The obtained model was subsequently least-squares refined against $3 \mathrm{D}$ ED data imposing constraints on the aromatic rings and hydrogen positions. Additionally, geometrical restraints for other interatomic distances and for the planarity of the flat blocks of the molecules were imposed. A sensible drop in residual $R \mathbf{f r o m} 32.05 \%$ to $30.69 \%$ was obtained with the introduction of a rotational twin law that exchanges $\boldsymbol{a}$ 
and $\boldsymbol{c}$ (the diffracting volume of the twin individual was refined to $8 \%$ of the total). More details about structure determination and refinement are reported in Table S1.

The model obtained from 3 D ED data was finally refined by Rietveld method against powder XRD data. As the sample diffracts to low resolution (ca. 1.5 $\AA$ ) the molecules were treated as semi-rigid bodies, by means of geometrical restraints, while the torsion angles were allowed to refine freely, in analogy to the approach used for the refinement against the ED data. The refinement converged to $R_{\mathrm{wp}}=4.35 \%, R_{\mathrm{F}}=3.56 \%, R_{\mathrm{F}_{2}}=$ $4.86 \%$ without any significant modification (Figure 3 ). The final structural model is shown in Figure 4 and is available as a cif file in Cambridge Structural Database with Deposition Number 2082996.

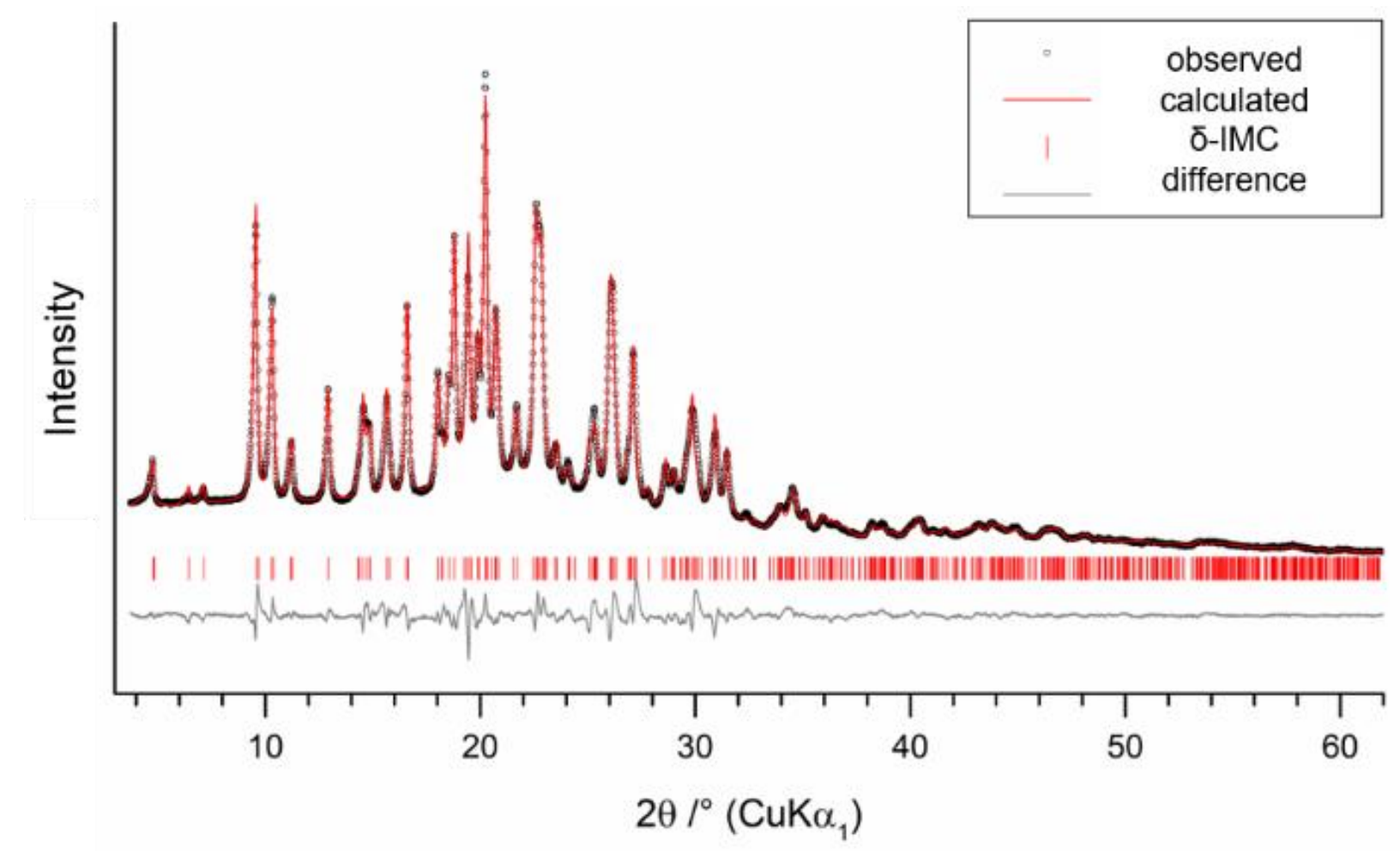

Figure 3. Final profile fit obtained after the restrained Rietveld refinement of the 3D ED structural model against powder XRD data. 

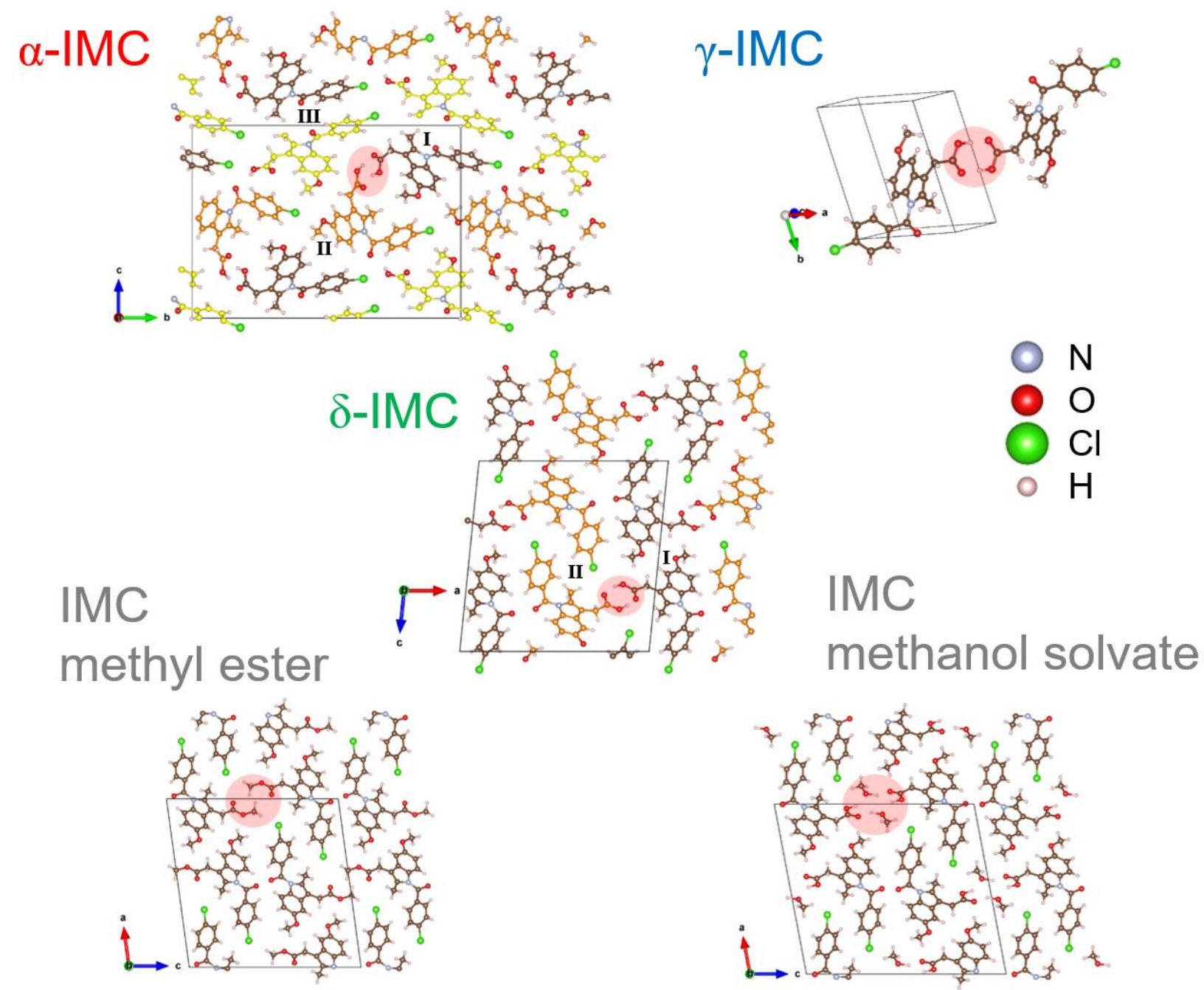

Figure 4. Structure models of $\alpha$-IMC, ${ }^{21} \gamma$-IMC, ${ }^{22} \delta$-IMC, indomethacin methyl ester ${ }^{48}$ and indomethacin methanol solvate. ${ }^{14}$ The carboxylic acid dimer is highlighted in red. For each model, carbon atoms are color-coded to distinguish the symmetry-independent molecules I (brown), II (orange) and III (yellow).

Structure description. The repeating motif in the structure of $\delta$-IMC is a dimer of molecules (I and II) connected by double hydrogen bonding through their carboxylic acid groups with $\mathrm{O}$... O distances 2.56o(9) and 2.844(9) Å. Molecules I and II adopt an almost specular conformation (Figure 5), but are not related by crystallographic symmetry, thus making the dimer and the overall structural packing noncentrosymmetric (Figure 4). The amidic and ether oxygens are involved in very weak $\mathrm{CH} \cdots \mathrm{O}$ interactions (with $\mathrm{H} \cdots \mathrm{O}$ distances in the range $2.387-2.878 \AA$ ). The chlorine atoms of both molecules point towards the dimeric carboxylic group ring motif, with the shortest $\mathrm{Cl}$... O distance of $2.974 \AA$ occurring between two copies of molecule II.

The carboxylic acid dimer is indeed a common motif in all forms of IMC. The supposed thermodynamically stable $\gamma$-IMC crystallizes in the centrosymmetric triclinic space group $P \overline{1}(2)$, with only one independent molecule in the asymmetric unit (Figure 4). Its crystal structure is stabilized by centrosymmetric hydrogenbonded ring motifs, formed between carboxylic groups ( $\mathrm{O} \cdots \mathrm{O}$ distance $2.651 \AA$ ). The amidic oxygen weakly 
interacts only with a $\mathrm{CH}$ from the methoxy group whereas the chlorine atom does not appear to interact with any other atom.

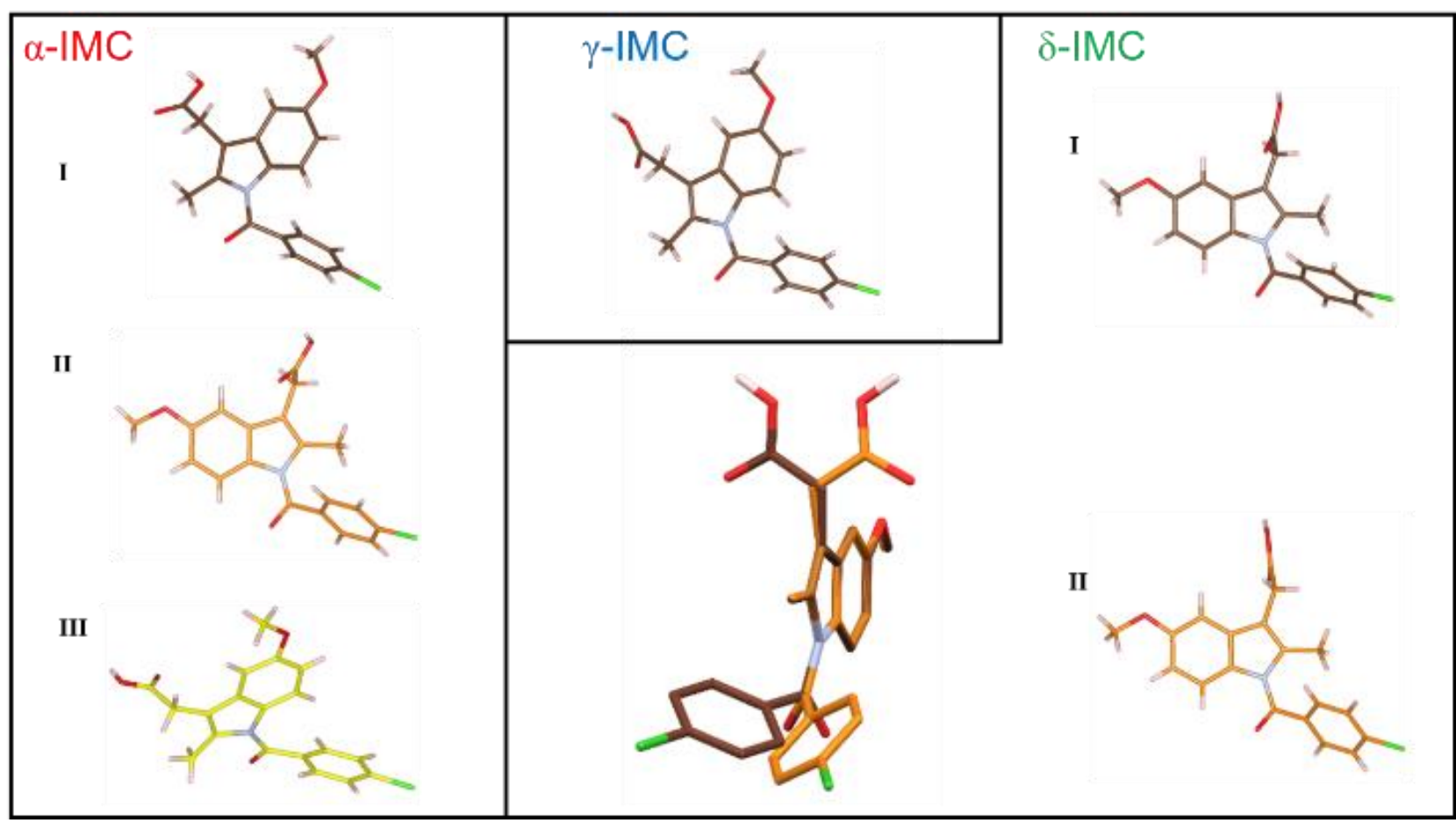

Figure 5. Distinct conformers present in $\alpha$-IMC, $\gamma$-IMC and $\delta$-IMC. For each compound, carbons of independent molecule I are in brown, carbons of molecule II are in orange and carbons of molecule III are in yellow. Atom colors as in Figure 4.

The 'metastable' $\alpha$-IMC crystallizes in the non-centrosymmetric monoclinic space group $P \mathbf{2}_{1}$, with three independent molecules in the asymmetric unit and a very different packing compared with $\gamma$-IMC (Figure 4). Molecules I and II form a dimer through their carboxylic groups (O...O distance 2.593 and 2.704 $\AA$, respectively). The carboxylic group of molecule III, instead, acts as a weak hydrogen-bond (H-bond) donor towards the amidic oxygen of molecule II (O...O distance $2.735 \AA$ ) and is relatively close to the chlorine atom of molecule I (Cl... O distance $2.958 \AA$ ).

In the solid state, the IMC molecule is found in different conformations (Figure 5). The most notable conformational differences are found around the linkage between the $p$-chlorobenzoyl group and the indole ring (torsion angle $\tau_{1}$ in Figure 1 ). The only molecule of $\gamma$-IMC and molecules I and III of $\alpha$-IMC are rather similar, with $\left|\tau_{1}\right| \approx 38^{\circ}, 28^{\circ}$ and $29^{\circ}$, respectively. Conversely, molecule II of $\alpha$-IMC and both molecules of $\delta$-IMC have a very distinct conformation, with $\left|\tau_{1}\right| \approx 156^{\circ}, 150^{\circ}$ and $155^{\circ}$, respectively.

Overall, cell parameters, crystal packing and molecular conformation of $\delta$-IMC are very similar to both indomethacin methyl ester ${ }^{48}$ and indomethacin methanol solvate ${ }^{14}$ (Figure 4 ). Nevertheless, the latter compounds crystallize in the centrosymmetric space group $P_{2_{1}} / n$, with only one independent molecule, forming centrosymmetric dimers involving the acid/ester groups. The similarity of $\delta$-IMC structure and indomethacin methanol solvate can be rationalized with $\delta$-IMC crystallization route, where the methanol solvate crystallizes first and then desolvates into $\delta$-IMC with minimal structural rearrangement. 
IR and Raman spectroscopy. Infrared (IR) spectroscopic data of $\delta$-IMC are well-documented in the literature, ${ }^{19,49-51}$ while Raman spectroscopic data have been reported only by Surwase et al. ${ }^{19}$ With the newfound knowledge of the crystal structure of $\delta$-IMC, these data can now be interpreted and offer a new perspective for understanding the structures of the remaining unsolved IMC polymorphs.
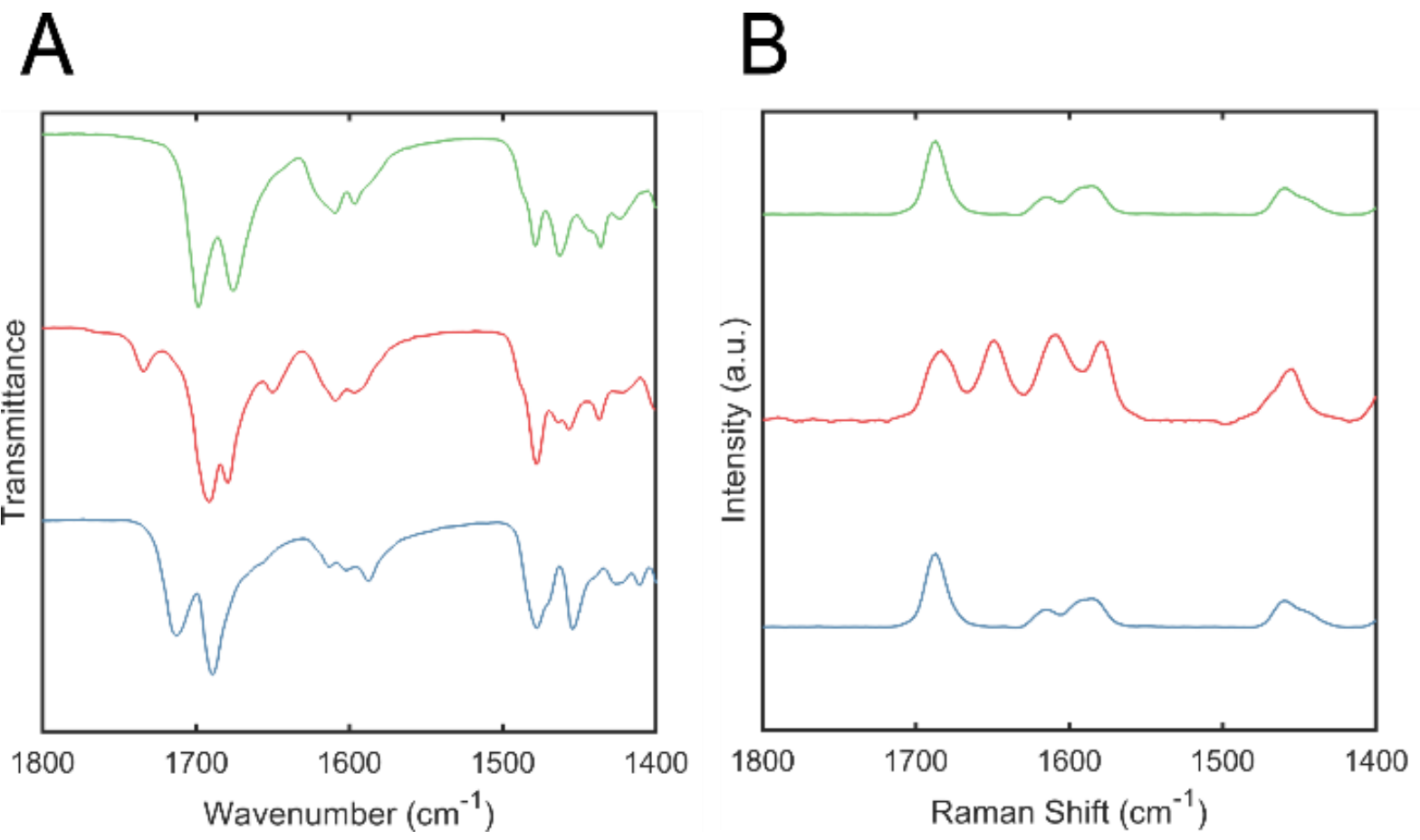

Figure 6. Vibrational data from (A) IR and (B) Raman spectra of the three solved forms of indomethacin: $\gamma$-IMC (blue), $\alpha$-IMC (red) and $\delta$-IMC (green).

New IR and Raman data for the $\alpha, \gamma$ and $\delta$-polymorphs of IMC are shown in Figure 6A and Figure 6B, respectively. Spectra are shown in the range $1800 \mathrm{~cm}^{-1}$ to $1400 \mathrm{~cm}^{-1}$ as the carbonyl stretches $(v(C=O))$, which are affected by IMC polymorphism, are typically observed in the $1700 \mathrm{~cm}^{-1}$ region..$^{50}$ IMC possesses two carbonyl groups, one belonging to an aromatic amidic group and the other to an aliphatic carboxylic acid group. Carboxylic acid stretch is active in the IR and occurs at a higher wavenumber than the aromatic amide carbonyl, which is both IR- and Raman-active. ${ }^{51}$ Therefore, the higher wavenumber peaks in Figure 6A can be assigned to the acid moiety and the lower wavenumber peaks to the amide carbonyl moiety. The presence of $\mathrm{H}$-bonding motifs on carbonyls can be inferred from the shift to lower wavenumbers of the peaks.

Assignments of carbonyl stretches for the three forms of IMC with known crystal structure are summarized in Table 1, as derived from the available structural models and comparison with previous interpretations in the literature. ${ }^{19}$ The asymmetric unit of $\gamma$-IMC contains only a single molecule which forms a carboxylic acid dimer. Thus, the carboxylic acid dimer stretch is related with the $1713 \mathrm{~cm}^{-1}$ peak, and the non-Hbonded amide carbonyl with the $1689 \mathrm{~cm}^{-1}$ peak in IR and with the $1698 \mathrm{~cm}^{-1}$ peak in Raman. 
Table 1. Assignments of carbonyl stretching bands, $v(\mathrm{C}=\mathrm{O})$, from experimental infrared (IR) and Raman (Ra) spectra for the structurally solved polymorphs $\alpha$-IMC, $\gamma$-IMC and $\delta$-IMC, together with the predicted assignments for the structurally unknown polymorphs $\varepsilon$-IMC, $\zeta$-IMC, $\eta$-IMC and $\tau$ IMC. ${ }^{19,20}$ All values are reported in $\mathrm{cm}^{-1}$.

\begin{tabular}{|c|c|c|c|c|c|c|c|}
\hline$v(\mathrm{C}=\mathrm{O})$ assignment & $\alpha$ & $\gamma$ & $\delta$ & $\varepsilon$ & $\zeta$ & $\eta$ & $\tau$ \\
\hline $\begin{array}{l}\text { Carboxylic acid } \\
\text { weakly or non-H-bonded }\end{array}$ & 1736 (IR) & & & & 1724 (IR) & 1728 (IR) & 1733 (IR) \\
\hline $\begin{array}{l}\text { Carboxylic acid } \\
\text { H-bonded }\end{array}$ & 1692 (IR) & 1713 (IR) & 1699 (IR) & 1711 (IR) & 1694 (IR, shoulder) & & 1696 (IR) \\
\hline $\begin{array}{l}\text { Amide } \\
\text { weakly or non-H-bonded }\end{array}$ & $\begin{array}{l}1680(\mathrm{IR}) \\
1685(\mathrm{Ra})\end{array}$ & $\begin{array}{l}1689 \text { (IR) } \\
1698(\mathrm{Ra})\end{array}$ & $\begin{array}{l}1675 \text { (IR) } \\
1689 \text { (Ra) }\end{array}$ & 1669 (IR) & $\begin{array}{l}1679(\mathrm{IR}) \\
1679(\mathrm{Ra})\end{array}$ & & 1976 (IR) \\
\hline $\begin{array}{l}\text { Amide } \\
\text { H-bonded }\end{array}$ & $\begin{array}{l}1652 \text { (IR) } \\
1650 \text { (Ra) }\end{array}$ & & & & $\begin{array}{l}1644 \text { (IR) } \\
1644 \text { (Ra) }\end{array}$ & $\begin{array}{l}1635 \text { (IR) } \\
1642 \text { (Ra) }\end{array}$ & \\
\hline
\end{tabular}

The asymmetric unit of $\alpha$-IMC contains three independent molecules, which exhibit distinct bonding motifs that affect the stretching frequencies and lead to the appearance of additional peaks (Figure 6). Molecules I and II form the common IMC motif, a carboxylic acid dimer which can be assigned to the 1692 $\mathrm{cm}^{-1}$ stretch, significantly lower than the $\gamma$-IMC carboxylic stretch, likely due to the less favorable dimer conformation. The carboxylic acid of molecule III, instead of dimerizing, forms a $\mathrm{H}$-bond $(\mathrm{OH} \cdots \mathrm{O})$ with the amide carbonyl of molecule II (2.08 Å) and the carboxylic acid carbonyl remains non-H-bonded. This $\mathrm{H}$-bonding motif results in a peak at $1736 \mathrm{~cm}^{-1}$ (molecule III carboxylic acid) and a peak at $1652 \mathrm{~cm}^{-1}$ in IR and at $165 \mathrm{om}^{-1}$ in Raman (molecule II amide carbonyl). The last peak for $\alpha$-IMC, at $168 \mathrm{o} \mathrm{cm}^{-1}$ in IR and at $1685 \mathrm{~cm}^{-1}$ in Raman, can be assigned to the non-H-bonded amide carbonyl groups of molecules I and III.

The $\delta$-IMC asymmetric unit is comprised of two molecules bonded through the common carboxylic acid dimer, which results in a carbonyl stretch at $1699 \mathrm{~cm}^{-1}$, again the lower wavenumber indicating a less favorable dimer conformation. Another single stretch is observed at $1675 \mathrm{~cm}^{-1}$ in IR and at $1689 \mathrm{~cm}^{-1}$ in Raman, consistent with the fact that both amide carbonyl groups of molecules I and II have only weak $\mathrm{H}$ bonding interactions. Remarkably, amide carbonyl of molecule I is at $2.495 \AA$ from an aromatic- $\mathrm{H}$ of the chlorophenyl termination of molecule II. In this perspective, the $\delta$-IMC structure can be described as a stacking of supramolecular chains, running parallel to $\boldsymbol{a}$ and connected by carboxylic acid dimers and chlorophenyl amide carbonyl moieties.

With this in mind, we can tentatively extend the interpretation of IR and Raman spectra reported by Surwase et al. ${ }^{19}$ for $\varepsilon$-IMC, $\zeta$-IMC and $\eta$-IMC and by Van Duong et al. ${ }^{20}$ for $\tau$-IMC (Table 1 ), noting that for $\varepsilon$-IMC and $\tau$-IMC only IR data have been reported.

There are two IR stretches obtained for $\varepsilon$-IMC in the region of $1700 \mathrm{~cm}^{-1}$. The stretch at $1711 \mathrm{~cm}^{-1}$ in IR is likely related to a carboxylic acid dimer, similar to the common motif observed for all structurally solved IMC forms. The stretch is particularly close to the corresponding peak observed for $\gamma$-IMC, suggesting it may possess comparable dimer conformations. The second peak is at $1669 \mathrm{~cm}^{-1}$ in IR and likely corresponds to a non-H-bonded amide carbonyl stretch. However, this value is notably lower than the one found for the corresponding peaks in $\gamma$-IMC and $\alpha$-IMC, and closer to the amide carbonyl peak observed for the newly solved structure of $\delta$-IMC. So, it can be suggested that the highly metastable $\varepsilon$-IMC possesses 
bonding motifs comparable to those present in $\delta$-IMC, concerning the combination of carboxylic acid dimers and chlorophenyl-amide carbonyl moieties.

In contrast, the measured spectra for $\zeta$-IMC contain peaks at either end of the active region, related to a non-H-bonded carboxylic acid carbonyl $\left(1724 \mathrm{~cm}^{-1}\right)$ and a H-bonded amide carbonyl $\left(1644 \mathrm{~cm}^{-1}\right)$, likely displaying the $(\mathrm{OH} \cdots \mathrm{O})$ bonding motif observed for $\alpha$-IMC. At the same time, the presence of weakly $\mathrm{H}-$ bonded or unbound amide carbonyl stretch is suggested by the peak at $1679 \mathrm{~cm}^{-1}$, together with the likely occurrence of carboxylic acid dimers, indicated by the shoulder at $1694 \mathrm{~cm}^{-1}$. Overall, the $\zeta$-IMC spectra are comparable with the $\alpha$-IMC spectra, however, the singular shape of the peak at $1694 \mathrm{~cm}^{-1}$ suggests the possibility of distinct packing with unique H-bonding motifs.

Unusually, the spectra of $\eta$-IMC possess exclusively the two peaks at the extremes of the carbonyl vibrational region, which suggests that the typical dimer motif is absent. Instead, $\eta$-IMC displays a unique $\mathrm{H}$-bonding motif with a weakly or non-H-bonded carboxylic acid carbonyl $\left(1728 \mathrm{~cm}^{-1}\right)$ and $\mathrm{H}$-bonded amide carbonyl (1625 $\mathrm{cm}^{-1}$ in IR and $1642 \mathrm{~cm}^{-1}$ in Raman), likely through a (OH...O) motif.

Finally, the IR spectra of $\tau$-IMC, suggests that this polymorph likely possesses a carboxylic acid dimer motif with a peak at $1696 \mathrm{~cm}^{-1}$, alongside molecule(s) with only weakly or non-H-bonded carboxylic acid carbonyl, with a peak at $1733 \mathrm{~cm}^{-1}$, and amide carbonyl, with a peak at $1976 \mathrm{~cm}^{-1}$.

Thermal analysis. Simultaneous synchrotron powder XRD-DSC is uniquely placed to directly reveal phase transformation on kinetically relevant timescales. This is particularly important for pharmaceuticals, as knowledge of relative stability is vital for judicious selection of polymorph. Heating from $40^{\circ} \mathrm{C}$ to $175{ }^{\circ} \mathrm{C}$ at a ramp rate of $10{ }^{\circ} \mathrm{C} / \mathrm{min}$ (Figure 7) showed that $\delta$-IMC underwent a solid-solid phase transition to $\alpha$ IMC in the temperature range $119-136^{\circ} \mathrm{C}\left(\mathrm{T}_{1}-\mathrm{T}_{2}\right)$. The extracted powder XRD patterns before and after the solid-solid phase transformation are assigned to the two forms (Figure $\mathrm{S}_{1}$ ). The pure phase $\alpha$-IMC remains stable in the temperature range $136-154{ }^{\circ} \mathrm{C}\left(\mathrm{T}_{2}-\mathrm{T}_{3}\right)$, followed by a melting event which has a temperature onset of $154{ }^{\circ} \mathrm{C}\left(\mathrm{T}_{3}\right)$ and is complete by $161{ }^{\circ} \mathrm{C}\left(\mathrm{T}_{4}\right)$. The thermogram of $\delta$-IMC and melting temperature of $\alpha$-IMC are both characteristic of their previously analyzed behavior. ${ }^{19,28}$ It is remarkable that $\delta$-IMC transforms to $\alpha$-IMC and not to $\gamma$-IMC, which is commonly referred to as the thermodynamically stable form. ${ }^{23}$ This evidence appears to support the claim by Ruggiero et al. ${ }^{24}$ that in fact $\alpha$-IMC is the more thermodynamically stable polymorph but is kinetically hindered during ambient temperature crystallization by the unfavorable conformation of molecule II. In this regard, conformers of $\delta$-IMC are very similar to molecule II of $\alpha$-IMC (Figure 5), and this similarity can drive or facilitate the solid-solid phase transition. However, $\delta$-IMC samples often include a small fraction of $\alpha$-IMC, which can act as seed during the transformation. 


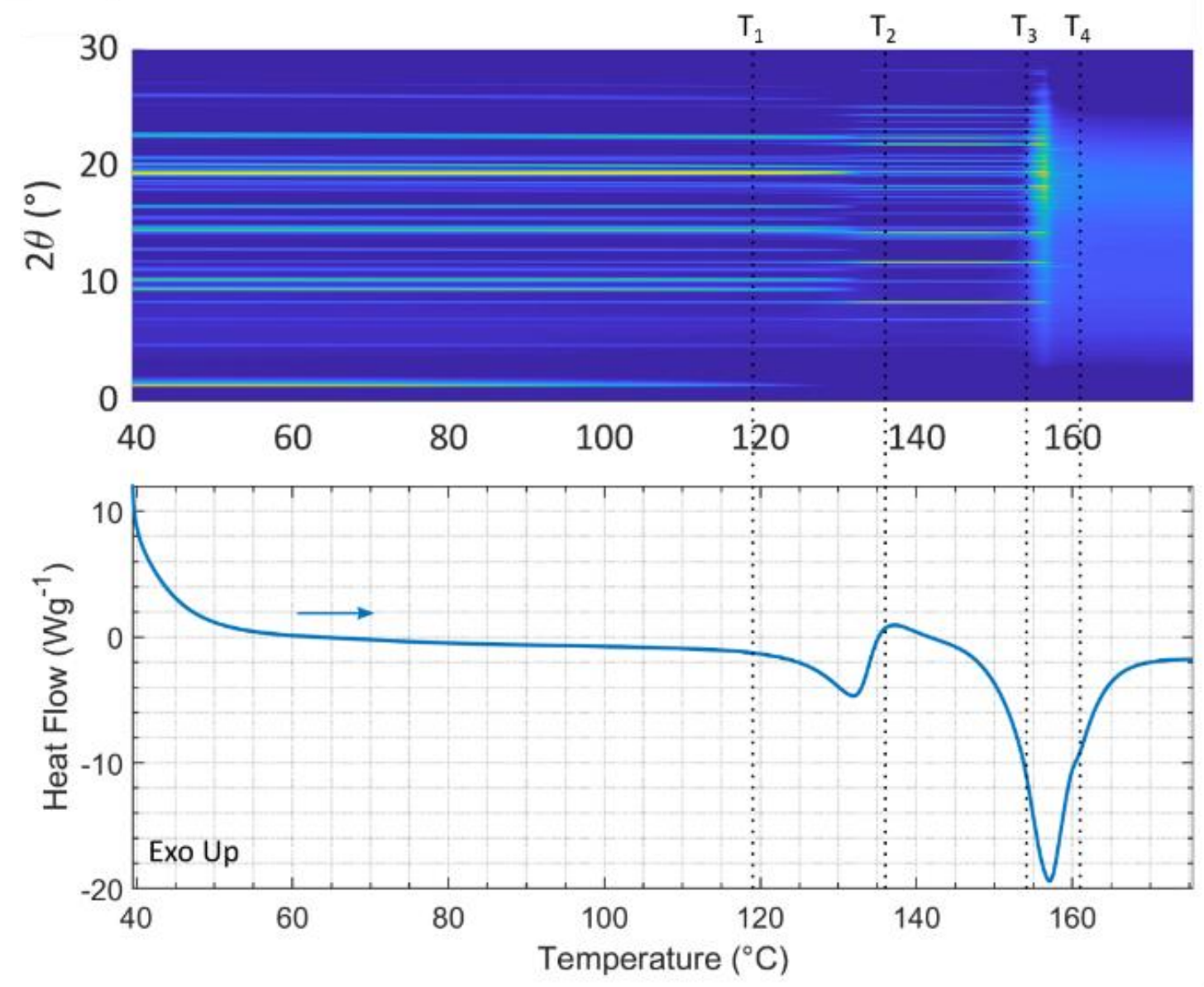

Figure 7. Synchrotron powder XRD-DSC result: (top) Surface plot of powder XRD patterns of $\delta$-IMC sample, during heating from $40-175^{\circ} \mathrm{C}$ and (bottom) the simultaneous DSC thermogram. $\delta$-IMC sample, containing a few of $\alpha$-IMC impurities, underwent a solid-solid transition (T1-T2) to pure $\alpha$-IMC (T2-T3), followed by a melting event $\left(\mathrm{T}_{3}-\mathrm{T}_{4}\right)$.

Dissolution Behavior. According to the Noyes-Whitney equation, the dissolution rate of a solid compound in a solvent is directly proportional to the solubility of the solid in the solvent and its specific surface area. ${ }^{52}$ To exclude an influence of the specific surface area on solubility, the various polymorphs were ground in a mortar and tablets were prepared with the resulting powder. ${ }^{13}$

The dissolution assay was first carried out in SGF, to determine the intrinsic solubility of each polymorph. The polymorphs $\alpha$-IMC, $\gamma$-IMC and $\delta$-IMC showed intrinsic solubility of 1.76, 2.11 and $17.89 \mu \mathrm{g} / \mathrm{mL}$, respectively. Thus, the solubility of the $\delta$-IMC polymorph was about 10 times the ones of both the $\alpha$-IMC and $\gamma$-IMC polymorphs.

Figure 8 shows the dissolution profiles for the different IMC polymorphs under study over the first 2 hours in SGF (Figure 8A) and the following 2 hours in SJF (Figure 8B). In all cases, compliance with sink conditions was verified. It is seen that the dissolution rate follows the same rank order as the solubility, i.e., $\alpha$-IMC $<\gamma$-IMC $<\delta$-IMC. However, the difference between the dissolution profiles of $\alpha$-IMC and $\gamma$-IMC is not significant, while the dissolution profile for $\delta$-IMC is indicative of a dissolution rate significantly higher than the one of both the $\alpha$-IMC and $\gamma$-IMC. In particular, it is observed that the percentage of $\delta$ IMC dissolved in 2 hours in SGF is about three times greater than the corresponding percentage of $\gamma$-IMC. This behavior may be related to the unusual proximity of chlorine atoms to carboxylic dimers, which likely affects the H-bonding. 

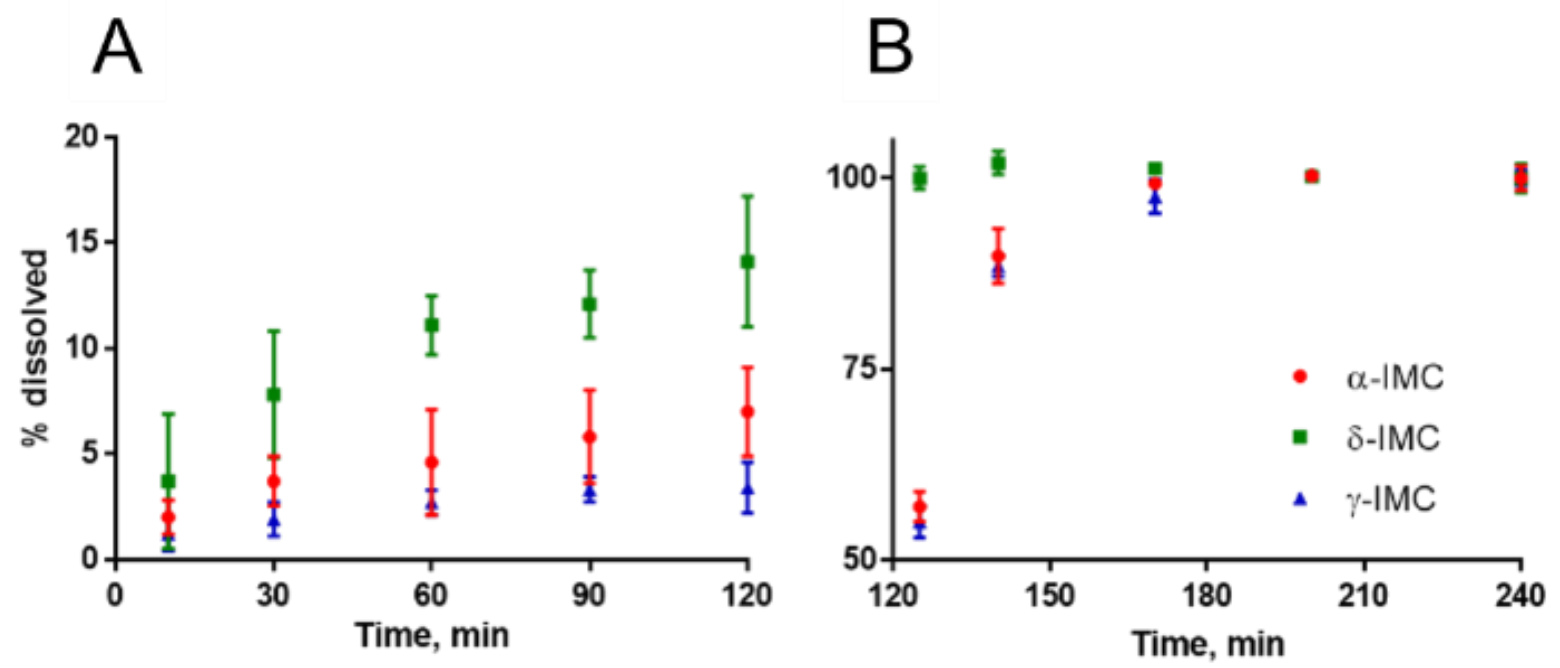

Figure 8. Dissolution profiles of $\alpha$-IMC, $\gamma$-IMC and $\delta$-IMC: (A) two hours in SGF and (B) the subsequent two hours in SJF. The mean values of three runs are shown along with their standard deviation.

Since IMC is a weak acid, its solubility at $\mathrm{pH} 6.8$ increases by two orders of magnitude due to the formation of the ionized species. ${ }^{33}$ Therefore, the dissolution reaches about $100 \%$ for all polymorphs after only 40 min in SJF. However, we observed that $\delta$-IMC was already completely dissolved after just 5 min, while at the same time only about $55 \%$ of the other polymorphs was dissolved (Figure $8 \mathrm{~B}$ ). A comparable result could be achieved only for the amorphous form of IMC, stabilized by using more sophisticated techniques, such as the liquisolid technique, ${ }^{54}$ the IMC dry coating technique ${ }^{55}$ and the application of chiral mesoporous silica carriers..$^{56}$

\section{CONCLUSIONS}

The crystal structure of $\delta$-indomethacin, a polymorph firstly reported almost 50 years ago ${ }^{28}$ but still poorly characterized, was solved thanks to the recent advances of the three-dimensional electron diffraction $(3 \mathrm{D}$ ED) method. ${ }^{29}$ Like many other active pharmaceutical ingredients (API), indomethacin appears in several polymorphic forms, which may have considerably different physical, chemical and medicinal properties. A large fraction of these pharmaceutical polymorphs exists only as tiny crystals with size of few micrometers, unsuitable for standard crystallographic techniques like single-crystal X-ray diffraction. In such cases, $3 \mathrm{D}$ ED proved a valid option for the structure characterization of single sub-micrometric grains. ${ }^{32-37}$ The main limit in the analysis of organic compounds is the fast deterioration induced by the electron beam. However, beam damage issues appear significantly mitigated after the introduction of single-electron detectors, able to get reasonable diffraction signal with an extremely mild electron dose rate. ${ }^{31,39}$

The structure solution of $\delta$-indomethacin allowed interpreting the available spectroscopic data and provided valuable information for the understanding of those polymorphs, whose structure is still unknown. ${ }^{19}$ Moreover, after heating we observed a direct solid-solid phase transition from $\delta$-indomethacin to $\alpha$-indomethacin, which is consistent with the similarity in their molecular conformation and opens new questions about the overall indomethacin phase diagram. Finally, although more in-depth stability analyses are needed, the results obtained from the present dissolution studies suggest that $\delta$-indomethacin is very soluble compared with other polymorphs and therefore appears particularly suitable for use in immediaterelease formulations.

The availability of efficient methods for structure characterization of poorly crystalline polymorphic forms is crucial for assessing the energy landscape of a given API, predicting its real potential for medicinal applications and possibly designing more efficient administration strategies. This is especially important 
when considering that $40 \%$ of marketed drugs and $75 \%$ of new candidates are poorly water-soluble and which represents a challenge for their bioavailability. ${ }^{57}$

\section{Author Contributions}

The manuscript was written through contributions of all authors.

\section{Notes}

The authors declare no competing financial interest.

\section{ACKNOWLEDGMENT}

I.A., A.E.L., E.M. and M.G. would like to thank Regione Toscana for funding the purchase of the ASI MEDIPIX detector through the FELIX project (POR CREO FERS 2014-2020) and Aldo Moscardini for inspiring discussions. S.R.H., V.H., J.P., and C.L.H. would like to acknowledge funding from the Engineering and Physical Sciences Research Council UK (Grants EP/Lo16648/1 and EP/Lo15544/1) and thank the Bristol Centre for Functional Nanomaterials, the Centre for Doctoral Training in Condensed Matter Physics and the MagnaPharm project, which has received funding from the European Union's Horizon 2020 Research and Innovation programme under Grant Agreement Number 73689. We thank Diamond Light Source for access to Beamline I12 under experiment MG25748, Dr Oxana Magdysyuk for her assistance during DSCXRD experiments, and TA Instruments (Waters, LLC) for donation of the Q2o DSC equipment.

\section{REFERENCES}

(1) Winter, C. A.; Risley, E. A.; Nuss, G. W. Anti-Inflammatory and Antipyretic Activities of Indomethacin. J. Pharmacol. Exptl. Therap. 1963, 141, 369-376.

(2) Hart, F. D.; Boardman, P. L. Indomethacin: A New Non-Steroid Anti-Inflammatory Agent. British Med. J. 1963, 2, 965-70.

(3) Lucas, S. The Pharmacology of Indomethacin. Headache 2016, 56, 436-446.

(4) Shen, T. Y.; Windholz, T. B.; Rosegay, A.; Witzel, B. E.; Wilson, A. N.; Willet, J. D.; Holtz, W. J.; Ellis, R. L.; Matzuk, A. R.; Lucas, S.; Stammer, C. H.; Holly, F. W.; Sarett, L. H.; Risley, E. A.; Nuss, G. W.; Winter, C. A. Non-Steroid Anti-Inflammatory Agents. J. Am. Chem. Soc. 1963, 85, 488-489.

(5) Nalamachu, S.; Wortmann, R. Role of Indomethacin in Acute Pain and Inflammation Management: A Review of the Literature. Postgraduate Medicine 2014, 126, 92-97.

(6) Amici, C.; Di Caro, A.; Ciucci, A.; Chiappa, L.; Castilletti, C.; Martella, V.; Decaro, N.; Buonavoglia, C.; Capobianchi, M. R.; Santoro, M. G. Indomethacin Has a Potent Antiviral Activity Against SARS Coronavirus. Antivir. Ther. 2006, 11, 1021-1030.

(7) Xu, T.; Gao, X.; Wu, Z.; Selinger, D. W.; Zhou, Z. Indomethacin Has a Potent Antiviral Activity Against SARS CoV-2 in vitro and Canine Coronavirus in vivo. 2020 https://doi.org/10.1101/2020.04.01.017624.

(8) Basavoju, S.; Boström, D.; Velaga, S. P. Indomethacin-Saccharin Cocrystal: Design, Synthesis and Preliminary Pharmaceutical Characterization. Pharm. Res. 2008, 25, 530-541.

(9) Majumder, M.; Buckton, G.; Rawlinson-Malone, C.; Williams, A. C.; Spillman, M. J.; Shankland, N.; Shankland, K. A Carbamazepine-Indomethacin (1:1) Cocrystal Prodused by Milling. CrystEngComm 2o11, 13, 6327-6328.

(10) Dudenko, D. V.; Williams, P. A.; Hughes, C. E.; Antzutkin, O. N.; Velaga, S. P.; Brown, S. P.; Harris, K. D. M. Exploiting the Synergy of Powder X-ray Diffraction and Solid-State NMR Spectroscopy in Structure Determination of Organic Molecular Solids. J. Phys. Chem. 2013, 117, 12258-12265.

(11) Connor, L. E.; Vassileiou, A. D.; Halbert, G. W.; Johnston, B. F.; Oswald, I. D. H. Structural Investigation and Compression of a Co-Crystal of Indomethacin and Saccharin. CrystEngComm 2019, 21, 4465-4472. 
(12) Wang, L.-Y.; Yu, Y.-M.; Jiang, F.-B.; Li, Y.-T.; Wu, Z.-Y.; Yan, C.-W. The First Zwitterionic Cocrystal of Indomethacin with Amino Acid Showing Optimized Physicochemical Properties as well as Accelerated Absorption and Slowed Elimination in vivo. New J. Chem. 2020, 44, 3930-3939.

(13) ElShaer, A.; Khan, S.; Perumal, D.; Hanson, P.; Mohammed, A.-R. Use of Amino Acid as Counterions Improves the Solubility of the BCS II Model Drug, Indomethacin. Current Drug Delivery 2011, 8, 363-372.

(14) Joshi, V.; Stowell, J. G.; Byrn, S. R. Solid-State Stability of Indomethacin Solvates. Mol. Cryst. Liq. Cryst. 1998, 313, 265-270.

(15) Hamdi, N.; Feutelais, Y.; Yagoubi, N.; Girolamode, D.; Legendre, B. Solvates of Indomethacin. J. Therm. Anal. Calorim. 2004, 76, 985-1001.

(16) McNamara, D. P.; Whitney, K. M.; Goss, S. L. Use of a Physiologic Bicarbonate Buffer System for Dissolution Characterization of Ionizable Drugs. Pharm. Res. 2003, 20, 1641-1646.

(17) Li, S.; Madan, P.; Lin, S. Effect of Ionization of Drug on Drug Solubilization in SMEDDS Prepared Using Capmul MCM and Caprylic Acid. Asian J. Pharm. Sci. 2017, 12, 73-82.

(18) O'Neil, M.J. The Merck Index - An Encyclopedia of Chemicals, Drugs, and Biologicals. Cambridge, UK: Royal Society of Chemistry, 2013; p. 922.

(19) Surwase, S. A.; Boetker J. P.; Saville, D.; Boyd, B. J.; Gordon, K. C.; Peltonen, L.; Strachan, C. J. Indomethacin: New Polymorphs of an Old Drug. Mol. Pharmaceutics 2013, 10, 4472-448o.

(20) Van Duong, T.; Lüdeker, D.; Van Bockstal, P.-J.; De Beer, T.; Van Humbeeck, J.; Van den Mooter, G. Polymorphism of Indomethacin in Semicrystalline Dispersions: Formation, Transformation, and Segregation. Mol. Pharmaceutics 2018, 15, 1037-1051.

(21) Chen, X.; Morris, K. R.; Griesser, U. J.; Byrn, S. R.; Stowell, J. G. Reactivity Differences of Indomethacin Solid Forms with Ammonia Gas. J. Am. Chem. Soc. 2002, 124, 15012-15019.

(22) Kistenmacher, T. J.; Marsh, R. E. Crystal and Molecular Structure of an Antiinflammatory Agent, Indomethacin, 1-(p-chlorobenzoyl)-5-methoxy-2-methylindole-3-acetic Acid. J. Am. Chem. Soc. 1972, 94, 1340-1345.

(23) Legendre, B.; Feutelais, Y. Polymorphic and Thermodynamic Study of Indomethacin. J. Therm. Anal. Cal. 2004, 76, 255-264.

(24) Ruggiero, M. T.; Sutton, J. J.; Fraser-Miller, S. J.; Zaczek, A. J.; Korter, T. M.; Gordon, K. C.; Zeitler, J. A. Revisiting the Thermodynamic Stability of Indomethacin Polymorphs with Low-Frequency Vibrational Spectroscopy and Quantum Mechanical Simulations. Cryst. Growth Des. 2018, 18, 6513-6520.

(25) Hilfiker, R. Polymorphism: In the Pharmaceutical Industry. Weinheim, Germany: Wiley, 2006.

(26) Cruz-Cabeza, A. J.; Reutzel-Edens, S. M.; Bernstein, J. Facts and Fictions about Polymorphism. Chem. Soc. Rev. 2015, 44, 8619-8635.

(27) Gao, L.; Liu, G.; Ma, J.; Wang, X.; Zhou, L.; Li, X. Drug Nanocrystals: in vivo Performances. J. Controlled Release 2012, 160, 418-430.

(28) Borka, L. The Polymorphism of Indomethacin. New Modifications, their Melting Behavior and Solubility. Acta Pharm. Suec. 1974, 11, 295-303.

(29) Gemmi, M.; Mugnaioli, E.; Gorelik, T. E.; Kolb, U.; Palatinus, L.; Boullay, P.; Hovmöller, S.; Abrahams, J. P. 3D Electron Diffraction: The Nanocrystallography Revolution. ACS Cent. Sci. 2019, 5, 1315-1329.

(30) Nederlof, I.; Van Genderen, E.; Li, Y. W.; Abrahams, J. P. A Medipix Quantum Area Detector Allows Rotation Electron Diffraction Data Collection from Submicrometre Three-Dimensional Protein Crystals. Acta Crystallogr. 2013, D69, 1223-1230.

(31) Van Genderen, E.; Clabbers, M. T. B.; Das, P. P.; Stewart, A.; Nederlof, I.; Barentsen, K. C.; Portillo, Q.; Pannu, N. S.; Nicolopoulos, S.; Gruene, T.; Abrahams, J. P. Ab initio Structure Determination of Nanocrystals of Organic Pharmaceutical Compounds by Electron Diffraction at Room Temperature Using a Timepix Quantum Area Direct Electron Detector. Acta Crystallogr. 2016, A72, 236-242.

(32) Das, P.P.; Mugnaioli, E.; Nicolopoulos, S.; Tossi, C.; Gemmi, M.; Galanis, A.; Borodi, G.; Pop, M. M. Crystal Structures of Two Important Pharmaceuticals Solved by 3D Precession Electron Diffraction Tomography. Org. Proc. Res. Dev. 2018, 22, 1365-1372.

(33) Gruene, T.; Wennmacher, J. T. C.; Zaubitzer, C.; Holstein, J. J.; Heidler, J.; Fecteau-Lefebvre, A.; De Carlo, S.; Müller, E.; Goldie, K. N.; Regeni, I.; Li, T.; Santiso-Quinones, G.; Steinfeld, G.; Handschin, S.; Van Genderen, E.; Van Bokhoven, J. A.; Clever, G. H.; Pantelic, R. Rapid Structure Determination of 
Microcrystalline Molecular Compounds Using Electron Diffraction. Angew. Chem. Int. Ed. 2018, 57, 1631316317.

(34) Jones, C. G.; Martynowycz, M. W.; Hattne, J.; Fulton, T. J.; Stoltz, B. M.; Rodriquez, J. A.; Nelson, H. M.; Gonen, T. The cryoEM Method MicroED as a Powerful Tool for Small Molecule Structure Determination. ACS Cent. Sci. 2018, 4, 1587-1592.

(35) Brázda, P.; Palatinus, L.; Babor, M. Electron Diffraction Determines Molecular Absolute Configuration in a Pharmaceutical Nanocrystal. Science 2019, 364, 667-669.

(36) Andrusenko, I.; Hamilton, V.; Mugnaioli, E.; Lanza, A.; Hall, C.; Potticary, J.; Hall, S. R.; Gemmi, M. The Crystal Structure of Orthocetamol Solved by 3D Electron Diffraction. Angew. Chem. Int. Ed. 2019, 131, $11035-11038$.

(37) Andrusenko, I.; Potticary, J.; Hall, S. R.; Gemmi, M. A New Olanzapine Cocrystal Obtained from Volatile Deep Eutectic Solvents and Determined by 3D Electron Diffraction. Acta Crystallogr. 202o, B76, 1036-1044.

(38) Crowley, K. J.; Zografi, G. Cryogenic Grinding of Indomethacin Polymorphs and Solvates: Assessment of Amorphous Phase Formation and Amorphous Phase Physical Stability. J. Pharm. Sci. 2002, 91, $492-507$.

(39) Lanza, A.; Margheritis, E.; Mugnaioli, E.; Cappello, V.; Garau, G.; Gemmi, M. IUCrJ 2019, 6, 178-188.

(40) Gemmi, M.; La Placa, M. G. I.; Galanis, A. S.; Rauch, E. F.; Nicolopoulos, S. Fast Electron Diffraction Tomography. J. Appl. Crystallogr. 2015, 48, 718-727.

(41) Gemmi, M.; Lanza, A. 3D Electron Diffraction Techniques. Acta Crystallogr. 2019, B75, 495-504.

(42) Palatinus, L.; Brázda, P.; Jelínek, M.; Hrdá, J.; Steciuk, G.; Klementová, M. Specifics of the Data Processing of Precession Electron Diffraction Tomography Data and their Implementation in the Program PETS2.o. Acta Crystallogr. 2019, B75, 512-522.

(43) Burla, M. C.; Caliandro, R.; Carrozzini, B.; Cascarano, G. L.; Cuocci, C.; Giacovazzo, C.; Mallamo, M.; Mazzone, A.; Polidori, G. Crystal Structure Determination and Refinement via SIR2014. J. Appl. Crystallogr. 2015, 48, 306-309.

(44) Groom, C. R.; Bruno, I. J.; Lightfoot, M. P.; Ward, S. C. The Cambridge Structural Database. Acta Crystallogr. 2016, B72, 171-179.

(45) Sheldrick, G. M. Crystal Structure Refinement with SHELXL. Acta Crystallogr. 2015, C71, 3-8.

(46) Petříček, V.; Dušek, M.; Palatinus, L. Crystallographic Computing System JANA2oo6: General features. Z. Kristallogr. 2014, 229, 345-352.

(47) Hart, M. L.; Drakopoulos, M.; Reinhard, C.; Connolley, T. Complete Elliptical Ring Geometry Provides Energy and Instrument Calibration for Synchrotron-Based Two-Dimensional X-ray Diffraction. J. Appl. Crystallogr. 2013, 46, 1249-1260.

(48) Trask, A. V.; Shan, N.; Jones, W.; Motherwell, W. D. S. Indomethacin Methyl Ester. Acta Crystallogr. 2004, E60, 508-509.

(49) Taylor, L. S.; Zografi, G. Spectroscopic Characterization of Interactions between PVP and Indomethacin in Amorphous Molecular Dispersions. Pharm. Res. 1997, 14, 1691-1698.

(50) Savolainen, M.; Heinz, A.; Strachan, C.; Gordon, K. C.; Yliruusi, J.; Radesb, T.; Sandler, N. Screening for Differences in the Amorphous State of Indomethacin Using Multivariate Visualization. Eur. J. Pharm. Sci. 2007, 30, 113-123.

(51) Strachan, C. J.; Rades, T.; Gordon, K. C. A Theoretical and Spectroscopic Study of Gamma-Crystalline and Amorphous Indomethacin. J. Pharm. Pharmacol. 2010, 59, 261-269.

(52) Dokoumetzidis, A.; Macheras, P. A Century of Dissolution Research: From Noyes and Whitney to the Biopharmaceutics Classification System. Int. J. Pharm. 20o6, 321, 1-11.

(53) Nokhodchi, A.; Javadzadeh, Y.; Siahi-Shadbad, M. R.; Barzegar-Jalali, M. The Effect of Type and Concentration of Vehicles on the Dissolution Rate of a Poorly Soluble Drug (Indomethacin) from Liquisolid Compacts. J. Pharm. Pharm. Sci. 2005, 8, 18-25.

(54) Javadzadeh, Y.; Siahi, M. R.; Asnaashari, S.; Nokhodchi, A. Liquisolid Technique as a Tool for Enhancement of Poorly Water-Soluble Drugs and Evaluation of their Physicochemical Properties. Acta Pharm. 2007, 57, 99-109.

(55) Tay, T.; Morton, D. A. V.; Gengenbach, T. R.; Stewart, P. J. Dissolution of a Poorly Water-Soluble Drug Dry Coated with Magnesium and Sodium Stearate. Eur. J. Pharm. Biopharm. 2012, 8o, 443-52. 
(56) Li, J.; Xu, L.; Yang, B.; Bao, Z.; Pan, W.; Li, S. Biomimetic Synthesized Chiral Mesoporous Silica: Structures and Controlled Release Functions as Drug Carrier. Mater. Sci. Eng. C Mater. Biol. Appl. 2015, 55, 367-372.

(57) Williams, H. D.; Trevaskis, N. L.; Charman, S. A.; Shanker, R. M.; Charman, W. N.; Pouton, C. W.; Porter, C. J. Strategies to Address Low Drug Solubility in Discovery and Development. Pharmacol. Rev. 2013, 65, 315-499. 


\section{Supporting Information}

Table S1. Selected crystallographic parameters from structure solution (SIR2014), and refinement based on the 3D ED data (SHELXL) and powder XRD (Jana2oo6).

\begin{tabular}{|c|c|}
\hline \multicolumn{2}{|l|}{ Crystallographic information } \\
\hline Asymmetric unit content & $\mathrm{C}_{38} \mathrm{H}_{32} \mathrm{~N}_{2} \mathrm{O}_{8} \mathrm{Cl}_{2}$ \\
\hline$Z$ & 4 \\
\hline Space group & $P_{2_{1}}$ \\
\hline \multicolumn{2}{|l|}{ Unit cell (from powder XRD data) } \\
\hline$a(\AA)$ & $18.3195(5)$ \\
\hline$b(\AA)$ & $5.09993(9)$ \\
\hline$c(\AA)$ & $18.5386(5)$ \\
\hline$\alpha\left(^{\circ}\right)$ & 90 \\
\hline$\beta\left(^{\circ}\right)$ & $95 \cdot 771(2)$ \\
\hline$\gamma\left({ }^{\circ}\right)$ & 90 \\
\hline Volume $(\AA 3)$ & $1723.25(6)$ \\
\hline \multicolumn{2}{|c|}{ Structure solution parameters (SIR2014 on $3 D$ ED data) } \\
\hline Data resolution $(\AA)$ & 1.0 \\
\hline No. of sampled reflections & 8982 \\
\hline No. of independent reflections & 3130 \\
\hline Independent reflections coverage (\%) & 81 \\
\hline Global thermal factor $U_{\text {iso }}\left(\AA^{2}\right)$ & 0.15712 \\
\hline$R_{\text {int }}(F)(\%)$ & $35 \cdot 92$ \\
\hline$C F(\%)$ & 53.038 \\
\hline \multicolumn{2}{|c|}{ Structure refinement parameters (SHELXL on ${ }_{3} D E D$ data) } \\
\hline Data resolution $(\AA)$ & 1.0 \\
\hline$R_{\text {int }}\left(F^{2}\right)(\%)$ & 34.49 \\
\hline No. of reflections (all) & 2925 \\
\hline No. of reflections $(>4 \sigma)$ & 1525 \\
\hline$R 1$ (all) (\%) & $37 \cdot 77$ \\
\hline
\end{tabular}




\begin{tabular}{|l|c|}
\hline$R_{1}(>4 \sigma)(\%)$ & 30.69 \\
\hline Goodness-of-fit & 2.726 \\
\hline \multicolumn{2}{|l|}{} \\
\hline \multicolumn{2}{|l|}{ Rietveld refinement parameters (Janazoo6 on powder XRD data) } \\
\hline $2 \theta$ range measured / step $\left(^{\circ}\right)$ & $2.000-61.985 / 0.015$ \\
\hline $2 \theta$ range used $\left(^{\circ}\right)$ & $3.725-61.985$ \\
\hline Data resolution $(\AA)$ & 1.5 \\
\hline No. of points & 4000 \\
\hline Parameters / restraints / constraints & $100 / 43 / 25$ \\
\hline$R_{\mathrm{wp}}(\%)$ & 4.35 \\
\hline$R_{\mathrm{F}}(\%)$ & 3.56 \\
\hline$R_{\mathrm{F} 2}(\%)$ & 4.86 \\
\hline
\end{tabular}

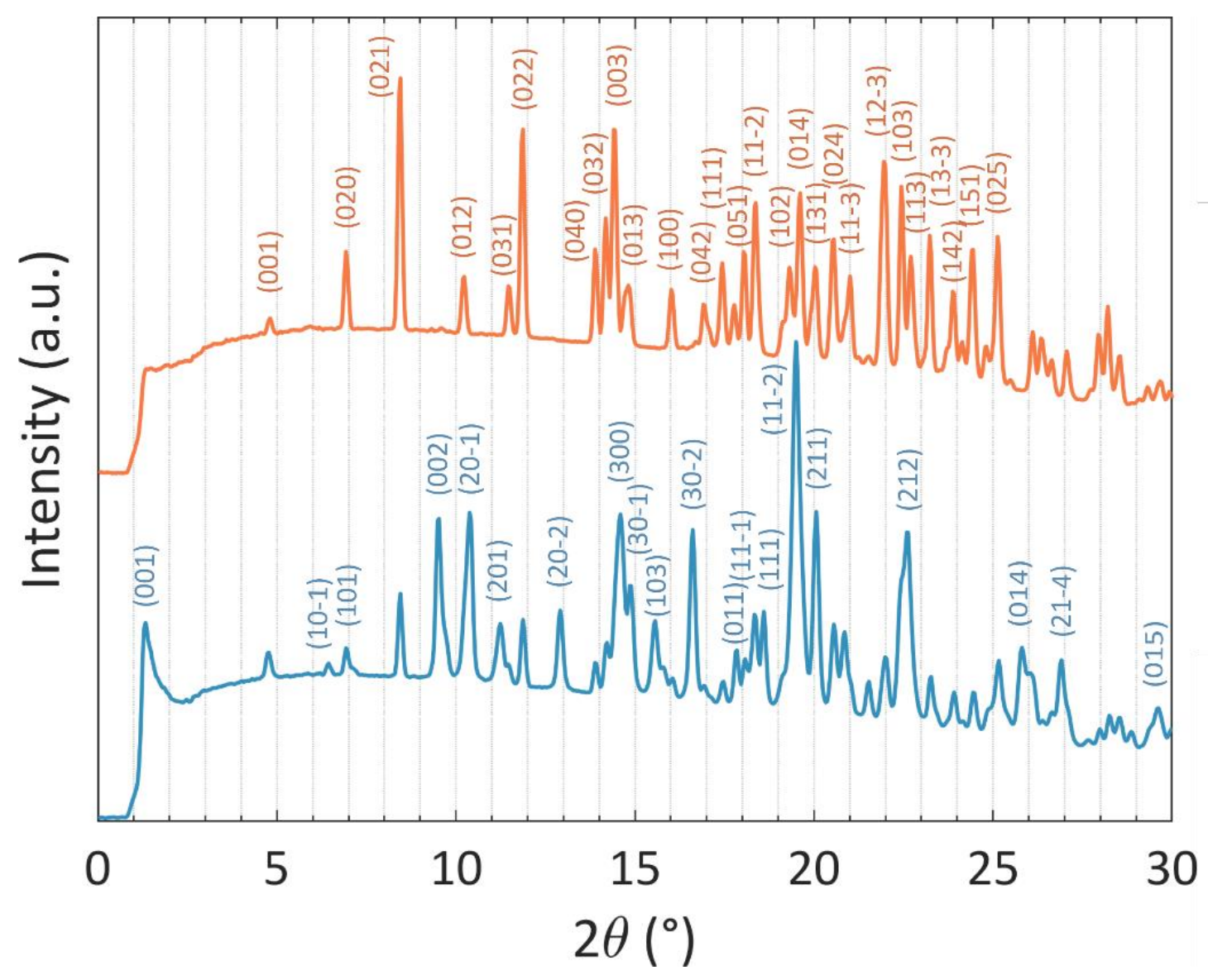

Figure S1. Extracted powder XRD patterns, before and after thermally induced phase transformation from $\delta$-IMC (blue), with some $\alpha$-IMC impurity, to pure phase $\alpha$-IMC (orange). Peaks are assigned from a simulated powder XRD pattern of the newly solved $\delta$-IMC and of $\alpha$-IMC obtained from the Cambridge Structural Database (CCDC-INDMETo2). ${ }^{21,44}$ 